\title{
Complete surgical resection of giant fibroma of the interventricular septum and left ventricle in an infant
}

Giovanni Battista Luciani, MD, ${ }^{\mathrm{a}}$ Stiljan Hoxha, MD, ${ }^{\mathrm{a}}$ Mara Pilati, MD, ${ }^{\mathrm{b}}$ Camilla Sandrini, MD PhD, Antonio Segreto, $\mathrm{MD},{ }^{\mathrm{a}}$ and Giuseppe Faggian, $\mathrm{MD},{ }^{\mathrm{a}}$ Verona, Italy

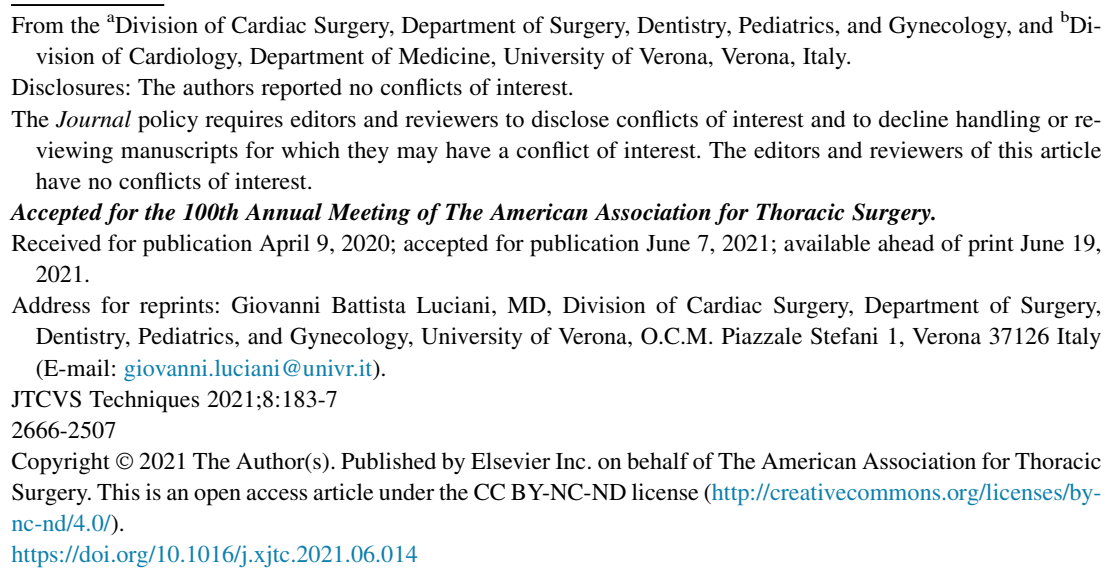
Video clip is available online.

Ventricular fibroma is the second most common benign cardiac tumor in children, yet responsible for significant morbidity and mortality. Surgical management of fibroma in infants and children is controversial, including resection ${ }^{1}$ (often partial ${ }^{1,2}$ ), single-ventricle palliation, or transplantation. ${ }^{3,4}$ We present a symptomatic infant with giant fibroma involving the interventricular septum (IVS) and the left ventricle (LV) who underwent complete surgical excision.

Consent for publication of this report was obtained from the parents of the patient. A 5-month old, 5.4-kg infant with failure to thrive, cyanosis, and tachypnea presented with cardiomegaly visible on chest radiograph, ventricular ectopy and runs of tachycardia apparent on electrocardiograph, and a giant mass infiltrating the IVS, anterolateral LV wall, and right ventricle conus visible on echocardiograph (Figure 1, $A$, and Video 1). Magnetic resonance imaging (Figure 1, B) and computed tomography (Figure 2) confirmed a $5.0 \times 5.8 \mathrm{~cm}$ mass suggesting fibroma. (Figure $1, C$ ). To accurately plan surgery, coronary angiogram (Figure 1,C) and 3-dimensional reconstruction of the heart were performed detailing the volume occupied by the mass and its relationship with the IVS, the left anterior descending artery (LAD), and the circumflex coronary

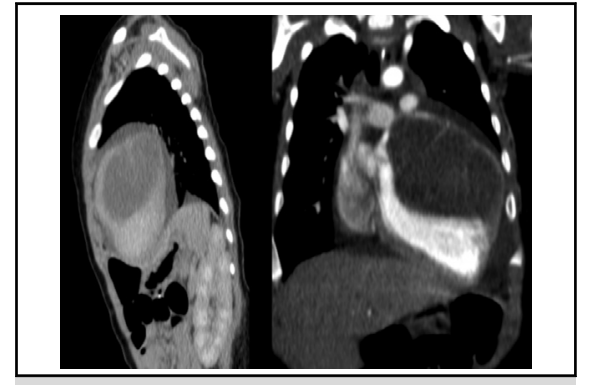

Cardiac CT scan in sagittal and coronal view showing a giant mass occupying the LV and IVS.

\section{CENTRAL MESSAGE \\ Radical excision of giant cardiac fibromas involving the IVS and LV are feasible even in very young infants. Strategic surgical plan- ning using advanced imaging and $3 \mathrm{D}$ reconstruction is fundamental.}

arteries (Figure 1,D). Via sternotomy, using hypothermic cardiopulmonary bypass after aortic crossclamping and cardioplegic arrest, the heart could be mobilized and the mass exposed. The epicardium of the anterolateral portion of the $\mathrm{LV}$ was incised, from base to apex, $2 \mathrm{~cm}$ lateral to the $\mathrm{LAD}$ (Figure 3, A). A firm, whitish, lobulated mass was gradually exposed by blunt and sharp dissection, taking great care to preserve all ventricular muscle on the septal and free wall sides. The septal branches of the LAD were dissected and clipped when found to be feeding the tumor (Figure 3, A). Full exposure of the huge mass required splitting into $3 \mathrm{seg}$ ments to achieve radical excision (Figure $3, B$ ). The tumor weighed $73 \mathrm{~g}$, twice the weight of a normal 5-month-old heart. Mass removal left a giant cavity in the thickness of the IVS and free wall. The right ventricle and LV septal layers were explored to rule out defects. Thereafter, the heart was repaired by approximating the layers with interrupted sutures completed by running suture of the LV free wall. Weaning from bypass required extracorporeal membrane oxygenation support due to episodes of sustained 


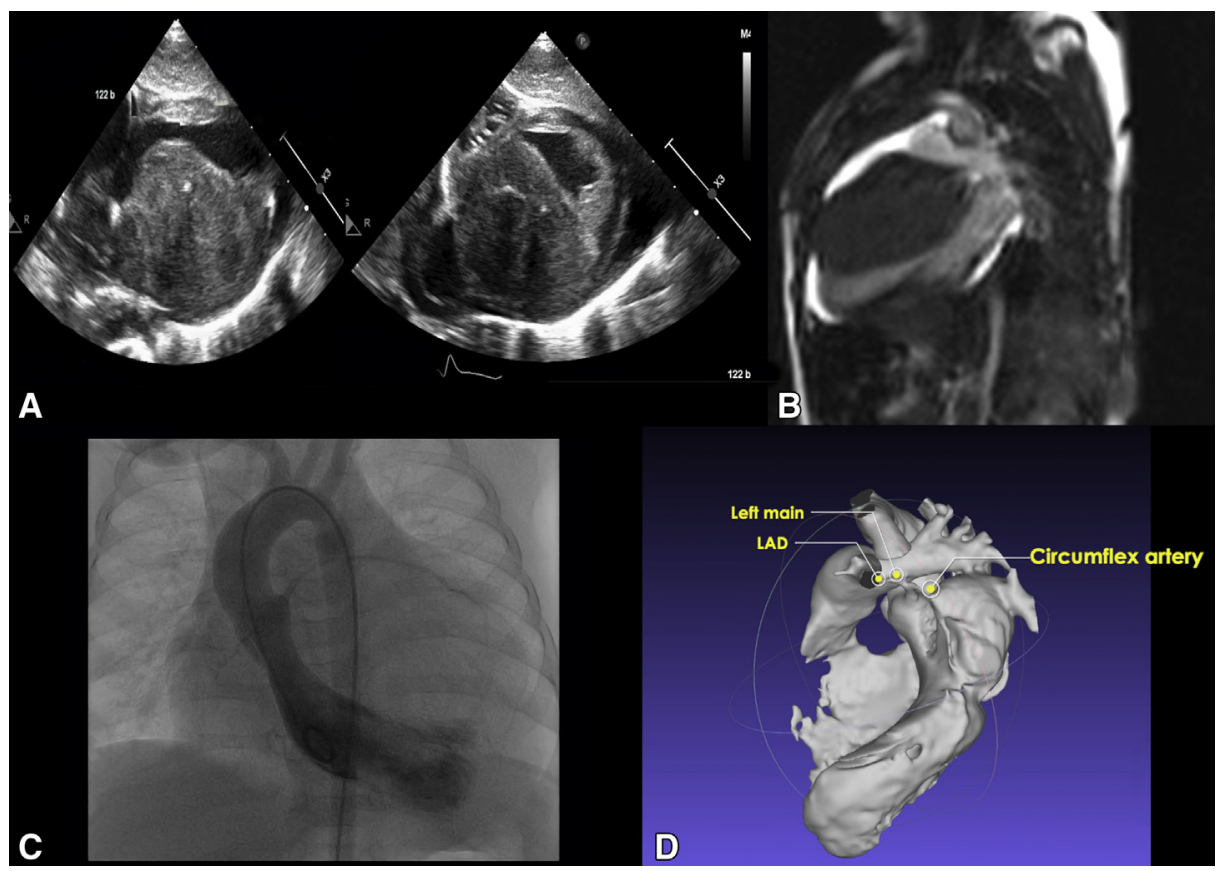

FIGURE 1. A, Short-axis view of preoperative transthoracic echocardiogram showing mass volume and extent of left ventricular (LV) and interventricular septum involvement. B, Sagittal view of cardiac magnetic resonance imaging detailing mass dimensions and interference with LV anatomy and function. C, Anteroposterior view of LV angiogram, confirming marked reduction of LV cavity volume and narrowing of LV outflow tract. Cranial dislocation of the left main stem and left anterior descending coronary artery caused by the giant mass is also apparent. D, Three-dimensional reconstruction of the heart and coronary arteries from cardiac magnetic resonance imaging. The topography and volume of the mass are evidenced by subtraction as a cavity. The exact position of the coronary arteries relative to the mass (cavity) is mapped and the presence of right ventricular and LV layers, although thin, of the interventricular septum are confirmed, suggesting feasibility of cleavage from the septum. $L A D$, Left anterior descending artery.

ventricular tachycardia. After echocardiographic evidence of severely depressed LV function, recovery allowed extracorporeal membrane oxygenation weaning on the third postoperative day, transfer from intensive care unit on the 14th postoperative day, and discharge on the 24th postoperative day. Histology showed fibroblasts, dense collagen, focal necrosis, and calcifications compatible with fibroma (Figure 3, C). Eight months after surgery, the infant is

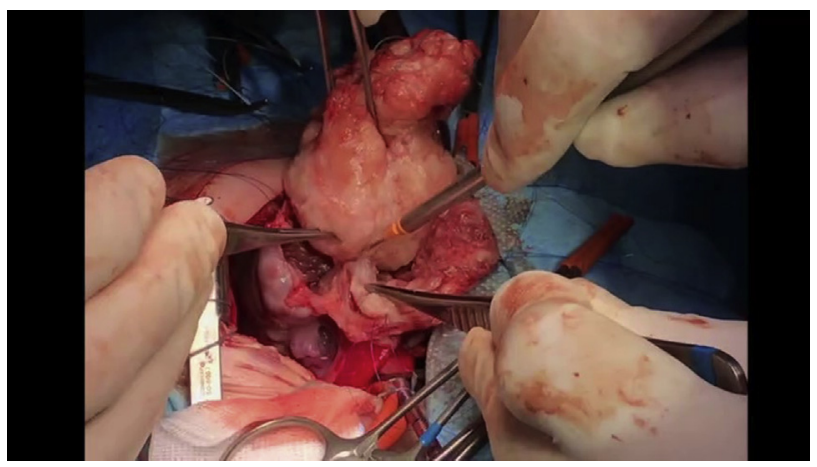

VIDEO 1. Complete case description, including preoperative diagnostic workup, operative procedure, and postoperative imaging, showing radical surgical excision of a giant interventricular septum and left ventricular free wall fibroma in an infant. Video available at: https://www.jtcvs.org/ article/S2666-2507(21)00410-7/fulltext. thriving with normalized cardiac silhouette visible on chest radiograph, no evidence of tumor relapse, and $40 \% \mathrm{LV}$ ejection fraction apparent on echocardiograph (Figure 3,D).

\section{DISCUSSION}

Consensus exists on surgical indication in children with symptomatic ventricular fibromas, although the ultimate management strategy is still undefined. Radical excision is often limited by tumor size and location, as well as age and size of the child. ${ }^{1}$ Alternative operations in the case of giant fibromas, especially in newborn or young infants, have included partial excision, ${ }^{1,2}$ single-ventricle palliation as bridge to excision, and transplantation.,

The case herein is 1 of 4 ever reported young infants undergoing excision of giant IVS and LV fibroma and the only with single-stage radical resection. ${ }^{1,2, \mathrm{E} 1}$ Whereas anecdotal survival after neonatal excision of LV fibroma has been documented, ${ }^{\mathrm{E} 1}$ this occurred at the expense of a 6-month-long hospitalization burdened by a series of life-threatening complications. A more cautious approach in young infants has consisted of partial excision, ${ }^{1,2}$ which may allow for faster postoperative ventricle functional recovery, but raises concern over relapse and the malignant ventricular arrhythmias typically associated with incomplete resection. ${ }^{5}$ In the present case, extent of IVS and LV free wall involvement 


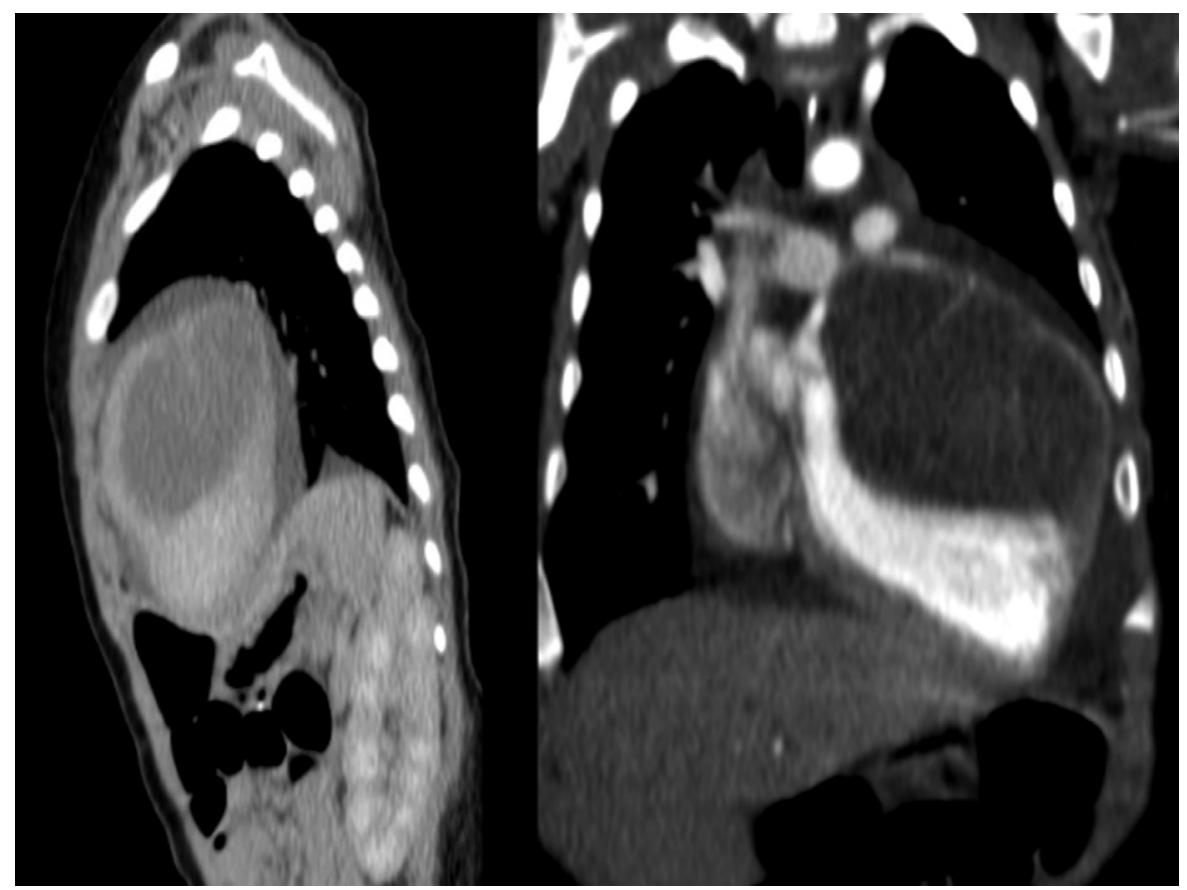

FIGURE 2. Cardiac computed tomography scan in sagittal and coronal view showing a giant mass occupying the left ventricle and interventricular septum.
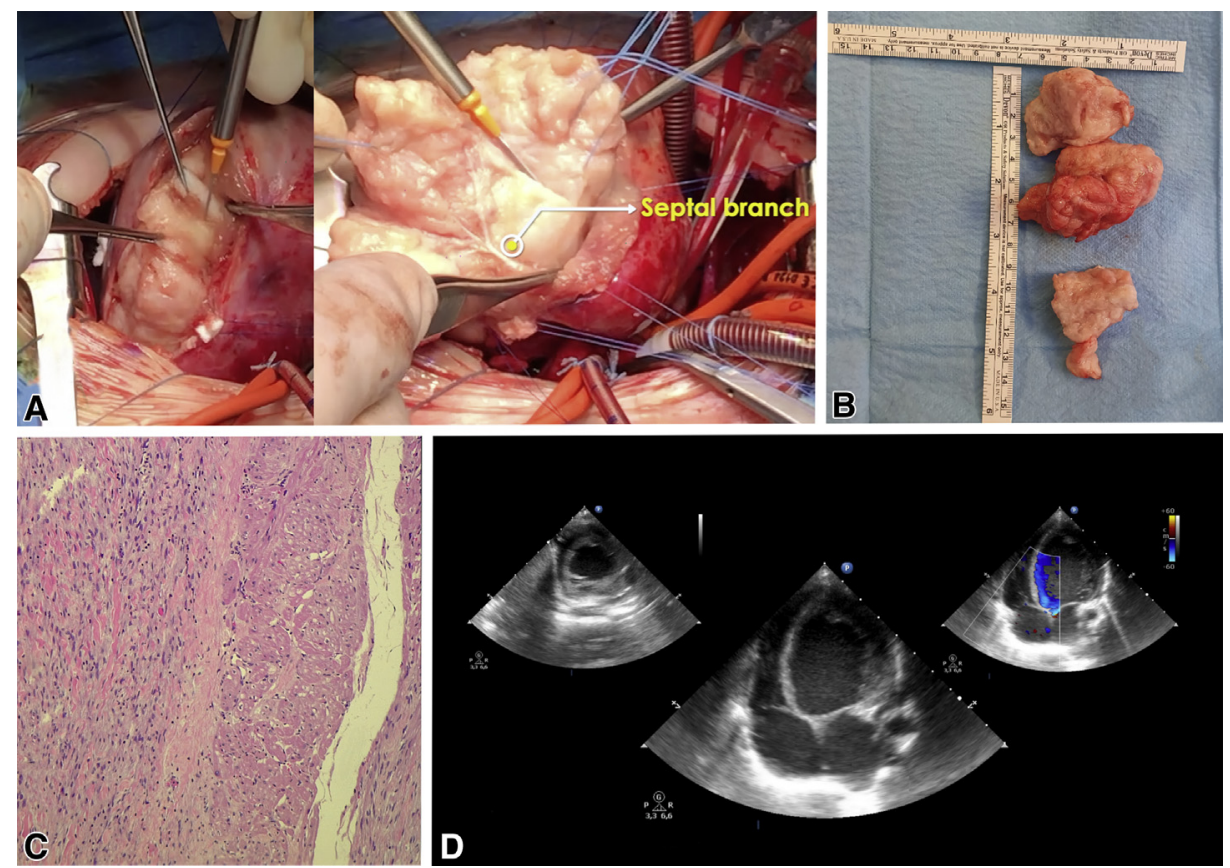

FIGURE 3. A, Operative view of the mass after longitudinal craniocaudal incision of the epicardium lateral to the left anterior descending, from cardiac base to apex. A firm, white, lobulated mass is evident and it appears distinct from a thin layer of anteroseptal and lateral myocardial tissue. Further exposure of the giant mass during surgery detailing the course of septal branches of the left anterior descending artery. Traction suture applied to the mass to aid cleavage from cardiac tissue is also evident. B, Gross anatomy of the mass, which was sectioned into 3 fragments to enhance exposure and complete radical excision. The dimensions of the mass are apparent. C, Microscopic anatomy of the tumor tissue with hematoxylin-eosin staining. Mesenchymal tissue with numerous spindle cells compatible with fibroblasts, dense collagen deposition, and microcalcifications. A layer of normal myocardial tissue is also evident. $\mathrm{D}$, Short axis and apical 4-chamber views of predischarge transthoracic echocardiograph, showing complete excision of the mass, recovered left ventricular and interventricular septum thickness, and absence of septal defects. 
was massive, as attested to by the tumor volume and weight. Advanced imaging with 3-dimensional reconstruction was combined with echocardiography and angiography (Figure 1), allowing us to plan, through virtual surgery, an operation respectful of vital anatomic structures dislodged by the mass. Key details were obtained, including the exact topography of LAD and circumflex coronary arteries and the feasibility of tumor cleavage from the IVS without entering ventricular cavities. Both aspects proved instrumental to enhanced ventricle functional recovery, although a brief period of mechanical circulatory support was necessary for electrical instability, as suggested by previous experience. ${ }^{1,5}$

\section{CONCLUSIONS}

Whether the potential for cardiomyocyte regeneration, greatest in young infants, may explain the dramatic ventricle functional recovery already apparent at discharge remains conjectural. We believe the present experience suggests that complete excision of extremely large cardiac fibromas involving the IVS and LV is both feasible and recommended even in young infants, possibly preventing late complications associated with incomplete excision or relapse. ${ }^{1,5}$

The authors thank Dr Elena Giulia Milano for generating 3-dimensional models of the heart from the computed tomography scans and Dr Eliana Gilioli for the histology images.

\section{References}

1. Nathan M, Fabozzo A, Geva T, Walsh E, del Nido PJ. Successful surgical management of ventricular fibromas in children. J Thorac Cardiovasc Surg. 2014;148: 2602-8.

2. Yörüker U, Yerebakan C, Mueller M, Akintürk H. Giant cardiac fibroma leading to cardiac arrest: surgical resection on the beating heart. J Thorac Cardiovasc Surg. 2015;150:e83-5.

3. Freire G, Jureidini S, Schowengerdt KO, Rahimi B, Tinker K, Fiore AC. Hybrid procedure for obstructive neonatal left ventricular tumors. Ann Thorac Surg. 2010;90:2057-9.

4. Kobayashi D, L'Ecuyer TJ, Aggarwal S. Orthotopic heart transplant: a therapeutic option for unresectable cardiac fibroma in infants. Congenit Heart Dis. 2012;7: E31-6.

5. Carreon CK, Sanders SP, Perez-Atayde AR, Del Nido PJ, Walsh EP, Geva T, et al. Interdigitating myocardial tongues in pediatric cardiac fibromas: plausible substrate for ventricular tachycardia and cardiac arrest. JACC Clin Electrophysiol. 2019;5:563-75. 


\section{E-Reference}

E1. Yabrodi M, Mastropietro CW, Darragh RK, Parent JJ, Ayres MD, Kean AC, et al. Management of complications caused by a massive left ventricle tumor in a neonate. Ann Thorac Surg. 2018;105:e259-61. 